\title{
Expectation of a collaboration between New Zealand and Japan for Developmental Origins of Health and Disease (DOHaD) research: a short introduction*
}

\section{Introductory remarks}

Sir Peter Gluckman, the former director of the Liggins Institute at the University Auckland, was invited to a special lecture at the 86th annual meeting of the Japan Endocrine Society in Sendai, Japan in April 2013. After the meeting, some council members of the Japan Society for Developmental Origins of Health and Disease (DOHaD-Japan) met him in Tokyo, Japan, and proposed a collaboration for $\mathrm{DOHaD}$ research between New Zealand and Japan. He agreed and suggested holding a kickoff meeting. During the 8th world congress of DOHaD 2013 in Singapore, 11 New Zealand and 14 Japanese $\mathrm{DOHaD}$ researchers met, developed mutual friendships and discussed potential collaborations. We initiated a number of collaborative research projects between the two countries and prepared grant applications for bilateral scientific programs between Japan and New Zealand for the Japan Society for the Promotion of Science (JSPS). The JSPS accepted our applications and agreed to financially support an international seminar at the Liggins Institute at the University of Auckland. 'New ZealandJapan joint seminar for $\mathrm{DOHaD}$ epigenetics and cohort research' was held at the Liggins Institute on 2-3 February 2016. At the beginning of the seminar, the council-general of Japan in Auckland, Mr Yoshitaka Yokoyama, addressed a special remark indicating not only the scientific significance, but also the important contribution of developing further friendships between the two countries. ${ }^{1}$ In total, 18 New Zealand and 14 Japanese researchers, including young postdoctoral researchers, presented their research data ${ }^{2}$ and discussed how to develop two-way research projects, including the exchange of young researchers, ${ }^{2}$ when the international symposium between New Zealand and Japan was proposed to be held during the 5 th annual meeting of DOHaD-Japan in Tokyo on 23-24 July 2016. Professor Frank Bloomfield and Professor Justin O'Sullivan came to Tokyo from New Zealand to present at the international symposium in addition to three Japanese speakers, and 180 Japanese $\mathrm{DOHaD}$ researchers attended. ${ }^{2}$ This theme issue was organized to introduce presentations at the international symposium.

\section{Promising future comparative study of $\mathrm{DOHaD}$ cohorts between New Zealand and Japan from the aspect of geographical and/or geopolitical characteristics}

Cohort studies play pivotal roles in the development of the DOHaD theory. ${ }^{3,4}$ There have been some pioneering cohort

* This work was presented at the international symposium of the 5th Annual Meeting of the Japan Society for the Developmental Origins of Health and Disease (DOHaD-Japan) in Tokyo on 23 July 2016. studies, that is, a British cohort study in the county of Hertfordshire (Barker Hypothesis), ${ }^{5-7}$ a Dutch cohort study following famine during World War II, ${ }^{8}$ and the Helsinki birth cohort study, ${ }^{9}$ which have led to the innovative concept that a relationship exists between environmental violence in early life and the incidence of non-communicable diseases (NCDs) throughout later life.

New Zealand and Japan are both composed of isolated islands, are located on the pan-Pacific side of the Asia Oceania region, and have similar total areas, that is, $268,000 \mathrm{~km}^{2}$ and $378,000 \mathrm{~km}^{2}$, respectively, with considerable mountain regions. However, different characteristics exist from geopolitical and ethnological aspects between the two countries, which may be attractive and promising targets for comparative studies of ongoing $\mathrm{DOHaD}$ cohort studies and/or the collaborative planning of new studies.

Based on 2015 data, the population of New Zealand is 4.7 million (17 persons $/ \mathrm{km}^{2}$ ), while that of Japan is 127.3 million (343 persons $/ \mathrm{km}^{2}$ ). Most Japanese residents are of a unique Mongoloid descent ${ }^{10}$ and have lived in the pan-Pacific islands of comparable area for thousands of years. Modern Japanese people have a unique food culture, Washoku, ${ }^{11}$ which differs from that of other Asian cultures, such as Chinese and Korean; however, there is now a considerable influence from a Western-style diet as well as food globalization. ${ }^{12}$

In Japan, the prevalence of obesity or being overweight has consistently increased among adult males as well as mature and elderly women, whereas undernourishment is common among young women of childbearing age because of their strong desire to be thin. ${ }^{13,14}$ A decrease in the body mass index of young Japanese women has been linked to an increase in low birth weight neonates as well as a decrease in the mean birth weight. ${ }^{14-16}$ Kubota et al. ${ }^{17}$ previously reported that mean energy intake by pregnant Japanese women was $<1600$ kilocalories/day through pregnancy, which is 30\% (second trimester) and 37\% (third trimester) below the recommendations of the Ministry of Health, Labour and Welfare, Japan, suggesting large numbers of relatively undernourished fetuses due to insufficient maternal energy intake. This is unusual in developed countries in which obesity is typically a serious social issue, even among pregnant women. Gluckman et al. ${ }^{16}$ indicated that insufficient energy intake by Japanese pregnant women may be related to the prevalence of NCDs from the viewpoint of the developmental origins hypothesis.

In contrast to the ethnicity of Japan, that of New Zealand is heterogeneous, comprising Caucasians, Polynesians, such as 
native Māori, Mongoloids, and considerable mixed ethnicity populations. The number of immigrants to New Zealand markedly increased, particularly from Europe, in the late 18th century, and this was followed by an increase in Asian immigrants, while Māori people reached New Zealand much earlier. Since two-thirds of the entire population of New Zealand is Caucasian, mostly European, a Western-style diet is the most popular. The prevalence of obesity is a serious social issue in New Zealand, particularly among Māori and Pacific islanders. ${ }^{18,19}$

Interestingly, mean birth weight of Chinese in New Zealand is more than $3400 \mathrm{~g},{ }^{20}$ while that of Japanese in Japan is $<3000 \mathrm{~g},{ }^{21}$ even with similar Asian Mongoloid background.

Since marked differences exist in nutritional supply from early to later life between Japan and New Zealand, a comparison of the epigenetic data of cohort studies may be useful for investigating contradictory nutritional influences on epigenetic modifications associated with the incidence of NCDs. On the other hand, ethnic differences between the two countries may be advantageous for examining the contribution of genetic diversity to the developmental origins hypothesis. Moreover, a comparison of epigenetic as well as genetic cohort data between the two countries may lead to the identification of common epigenetic pathways underlying the $\mathrm{DOHaD}$ theory among individuals with a wide variety of genetic backgrounds.

\section{DOHaD research centers in New Zealand and Japan}

The Liggins Institute at the University of Auckland, New Zealand, has focused on improving life-long health through research into the long-term consequences of early life events, and is the leading $\mathrm{DOHaD}$ research center not only in New Zealand, but also worldwide. ${ }^{22}$ Although there is currently no single institution that leads $\mathrm{DOHaD}$ research in Japan, DOHaD-Japan was launched in July 2012. ${ }^{2}$ DOHaD-Japan became an official chapter member of the International Society for $\mathrm{DOHaD}$ in September 2014. DOHaD-Japan has held annual meetings, at which -200 Japanese researchers have attended every year from a wide variety of $\mathrm{DOHaD}$-associated research areas, such as birth cohort studies, developmental neurology, animal science, zootechnical science, toxicology, nutritional science, genetics, epigenetics, neonatology, obstetrics, pediatrics, internal medicine, midwifes and nutritionists. Thus, New Zealand and Japan both have core organizations, albeit different types, for organizing and continuously supporting collaborative projects on $\mathrm{DOHaD}$ research between the two countries.

\section{Conclusion}

Collaborative $\mathrm{DOHaD}$ research between New Zealand and Japan has just been launched. It will be beneficial for future collaborative $\mathrm{DOHaD}$ research to consider differences in life-long nutritional supply as well as ethnic diversity between the two countries under similar geographical backgrounds.

\section{Financial Support}

This work was supported in part by Grants-in-Aid for Scientific Research from the Ministry of Education, Science, Culture and Sports, Japan (No. 15H04882, No. 16K15703).

\section{References}

1. Council-General. 2016. Retrieved 21 August 2017 from http://www. auckland.nz.emb-japan.go.jp/culture/pastevent_list/past_events_j_ 20160202.html.

2. The Japan Society for Developmental Origins of Health and Disease (DOHaD-Japan). 2016; Retrieved 21 August 2017 from http://square.umin.ac.jp/Jp-DOHaD/J-DOHaD.html.

3. Fukuoka H. DOHaD (Developmental Origins of Health and Disease) and Birth Cohort Research. J Nutr Sci Vitaminol (Tokyo). 2015; 61(Suppl.), S2-S4.

4. Hanson M, Gluckman P. Commentary: developing the future: life course epidemiology, $\mathrm{DOHaD}$ and evolutionary medicine. Int J Epidemiol. 2016; 45, 993-996.

5. Barker DJ, Osmond C. Infant mortality, childhood nutrition, and ischaemic heart disease in England and Wales. Lancet. 1986; 1, 1077-1081.

6. Barker DJ, Winter PD, Osmond C, Margetts B, Simmonds SJ. Weight in infancy and death from ischaemic heart disease. Lancet. 1989; 2, 577-580.

7. Hales CN, Barker DJ. Type 2 (non-insulin-dependent) diabetes mellitus: the thrifty phenotype hypothesis. Diabetologia. 1992; 35, 595-601.

8. Roseboom TJ, Painter RC, van Abeelen AF, Veenendaal MV, de Rooij SR. Hungry in the womb: what are the consequences? Lessons from the Dutch famine. Maturitas. 2011; 70, 141-145.

9. Eriksson JG. Developmental Origins of Health and Disease - from a small body size at birth to epigenetics. Ann Med. 2016; 48, 456-467.

10. Shinka T, Tomita K, Toda T, et al. Genetic variations on the $\mathrm{Y}$ chromosome in the Japanese population and implications for modern human Y chromosome lineage. J Hum Genet. 1999; 44, 240-245.

11. Oikawa T, Uneyama H. WASHOKU and health: new approach with Dashi/Umami in the Medical and Nutritional Health Care. Yakugaku Zasshi. 2016; 136, 1325-1326.

12. Kusaka S, Ishimaru E, Hyodo F, et al. Homogeneous diet of contemporary Japanese inferred from stable isotope ratios of hair. Sci Rep. 2016; 6, 33122.

13. Hayashi F, Takimoto H, Yoshita K, Yoshiike N. Perceived body size and desire for thinness of young Japanese women: a population-based survey. Br J Nutr. 2006; 96, 1154-1162.

14. Itoh H, Kanayama N. Low birth weight and risk of obesity potential problem of Japanese people. Curr Women Health Rev. 2009; 5, 212-219.

15. Goto Y. Diseases in the 21st century. J Japan Soc Study Obes (Japanese). 2006; 12, 1-2.

16. Gluckman PD, Seng CY, Fukuoka H, Beedle AS, Hanson MA. Low birthweight and subsequent obesity in Japan. Lancet. 2007; 369, 1081-1082.

17. Kubota K, Itoh H, Tasaka M, et al. Changes of maternal dietary intake, bodyweight and fetal growth throughout pregnancy in pregnant Japanese women. J Obstet Gynaecol Res. 2013; 39, 1383-1390.

18. Claridge R, Gray L, Stubbe M, Macdonald L, Tester R, Dowell AC. General practitioner opinion of weight management 
interventions in New Zealand. J Prim Health Care. 2014; 6, 212-220.

19. Poppitt SD, Silvestre MP, Liu A. Etiology of obesity over the life span: ecologic and genetic highlights from New Zealand cohorts. Curr Obes Rep. 2014; 3, 38-45.

20. McCowan L, Stewart AW. Term birthweight centiles for babies from New Zealand's main ethnic groups. Aust NZ J Obstet Gynaecol. 2004; 44, 432-435.

21. Terada M, Matsuda Y, Ogawa M, Matsui H, Satoh S. Effects of maternal factors on birth weight in Japan. J Pregnancy. 2013; 2013, 172395, http://dx.doi.org/10.1155/2013/172395.
22. Kirby T. Profile: The Liggins Institute, New Zealand. Lancet. 2015; 386, 234.

H. Itoh and N. Kanayama Department of Obstetrics and Gynecology, Amamatsu University School of Medicine, Higashi-ku, Hamamatsu, Japan Email: hitou-endo@umin.ac.jp 